\title{
Protocolo operacional de extubação na unidade de terapia intensiva adulta de um hospital de ensino da região norte do estado do Ceará
}

RESUMO | Objetivo: analisar um Protocolo Operacional de Extubação na Unidade de Terapia Intensiva (UTI) Adulta de um Hospital de Ensino da região Norte do estado do Ceará. Método: Estudo longitudinal de caráter descritivo com abordagem quantitativa. A coleta de dados foi realizada por meio da aplicação de uma ficha de avaliação e um protocolo de extubação com sua aplicação e análise, no período de agosto a outubro de 2018, análise estatística se deu através do uso do teste qui-quadrado, ao nível de $5 \%$ de significância, a pesquisa foi aprovado pelo Comitê de Ética em Pesquisa Envolvendo Seres Humanos sob o número CAAE 89038418.6.0000.8109, sob o Parecer $N^{\circ}$ 2.749.337. Resultado: o uso de um protocolo de extubação resultou em uma redução das taxas de falha de extubação, diminuindo os índices que levam a estas falhas demonstrando a eficácia do mesmo. Conclusão: necessidade de novas pesquisas, abordando um número maior de indivíduos, uso de educação permanente para a equipe multiprofissional, com a aplicação de protocolos pela equipe.

Palavras-chaves: Extubação; Unidade de Terapia Intensiva; Fisioterapeuta.

ABSTRACT | Objective: to analyze an Operational Protocol for Extubation in the Adult Intensive Care Unit (ICU) of a Teaching Hospital in the northern region of the state of Ceará. Method: Longitudinal descriptive study with quantitative approach. Data collection was performed through the application of an evaluation form and an extubation protocol with its application and analysis, in the period from August to October 2018, statistical analysis was done using the chi-square test, at the level of $5 \%$ significance, the research was approved by the Research Ethics Committee Involving Human Beings under the number CAAE 89038418.6.0000.8109, under Opinion No. 2,749,337. Results: The use of an extubation protocol resulted in a reduction in the rates of extubation failure, reducing the indices that lead to these failures demonstrating the efficacy of extubation. Conclusion: need for new research, addressing a greater number of individuals, use of permanent education for the multiprofessional team, with the application of protocols by the team.

Keywords: Extubation; Intensive care unit; Physiotherapist.

RESUMEN | Objetivo: analizar un Protocolo Operativo de Extubación en la Unidad de Terapia Intensiva (UTI) Adulta de un Hospital de Enseñanza de la región Norte del estado de Ceará. Método: Estudio longitudinal de carácter descriptivo con abordaje cuantitativo. La recolección de datos fue realizada por medio de la aplicación de una ficha de evaluación y un protocolo de extubación con su aplicación y análisis, en el período de agosto a octubre de 2018, el análisis estadístico se dio a través del uso del test chi-cuadrado, al nivel de 5\% de significancia, la encuesta fue aprobada por el Comité de Ética en Investigación Envolviendo Seres Humanos bajo el número CAAE 89038418.6.0000.8109, bajo el Dictamen № 2.749.337. Resultado: el uso de un protocolo de extubación resultó en una reducción de las tasas de fallo de extubación, disminuyendo los índices que llevan a estas fallas demostrando la eficacia del mismo. Conclusión: necesidad de nuevas investigaciones, abordando un número mayor de individuos, uso de educación permanente para el equipo multiprofesional, con la aplicación de protocolos por el equipo.

Palabras claves: : Extubación; Unidad de terapia intensiva; Fisioterapeuta.

\section{lara Laís Lima de Sousa}

Fisioterapeuta. Especialista em Urgência e Emergência com caráter em Residência pela Santa Casa de Misericórdia de Sobral.

\section{Thaiane Vasconcelos Carvalho}

Farmacêutica. Residente Multiprofissional em Urgência e Emergência pela Santa Casa de Misericórdia de Sobral.

\section{Kauanny Gomes Gonçalves}

Enfermeira. Especialista em Urgência e Emergência com caráter em Residência pela Santa Casa de Misericórdia de Sobral.

\section{Francisco Mardônio Aguiar Cavalcante}

Fisioterapeuta. Especialista em Fisioterapia em Terapia Intensiva pela universidade de São Paulo.

\section{Dênis Frota Guimarães}

Fisioterapeuta. Mestre, Docente do Curso de Fisioterapia no Centro Universitário INTA (UNINTA).

\section{José Henrique Linhares}

Fisioterapeuta. Mestre, Docente do Curso de Fisioterapia no Centro Universitário INTA (UNINTA).

Recebido em: 04/03/2019

Aprovado em: 07/06/2019
INTRODUÇÃO

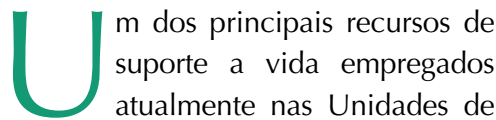
Terapia Intensiva (UTI) é a ventilação mecânica (VM), que se constitui como principal forma de tratamento a pacientes graves $^{1}$. Caracterizando-se como um método de suporte ventilatório indicado para terapêutica de usuários com insuficiência respiratória aguda ou crônica ${ }^{2}$. Podendo ser utilizada de forma não invasiva, a partir de uma interface externa, e de forma invasiva, através de um tubo endotraqueal ou cânula de traqueostomia ${ }^{3}$. Cujo principal 
objetivo consta em substituir ou auxiliar a função ventilatória do doente por meio de uma pressão positiva, quando se fizer necessário corrigir distúrbios que comprometem a ventilação ou as trocas gasosas ${ }^{4}$.

Entretanto, apesar de ser uma importante intervenção para pacientes sob terapia intensiva, a ventilação mecânica invasiva (VMI) está associada a diversas complicações, especialmente sob uso prolongado. Representando riscos de lesão traqueal, lesão pulmonar inerente à própria VMI, aumento da dependência ao ventilador, óbito, e sua complicação mais comum: a pneumonia 5 .

Portanto, quando conectado a uma prótese ventilatória, o paciente deve ser monitorizado com objetivo de identificar o momento propicio para o início do desmame da VM, pois quanto mais precoce a retirada, maiores serão as chances do processo de extubação ser bem-sucedido $^{6}$. A retirada gradual do suporte ventilatório (desmame) pode ser iniciada após o processo que desencadeou a necessidade da intubação traqueal e quando a VM se encontrar estabilizada ${ }^{7}$. Assim, conceitua-se a extubação como sendo a finalização deste processo, que ocorre com a retirada da via aérea artificial após atingidos os parâmetros que garantem a respiração espontânea definitiva ${ }^{8}$.

A fim de reduzir tais índices insatisfatórios, estudos tem demonstrado a importância do uso de protocolos baseados na avaliação clínica diária e identificação sistemática de pacientes em condições para interrupção do suporte ventilatório, que preconizam uma previsão acurada do desfecho da extubação e redução significativa da $\mathrm{VM}^{9}$. Observando-se na literatura, a inquietação na realização de indicadores preditivos descritos com o intuito de identificar os pacientes capazes de reassumirem a ventilação espontânea ${ }^{10,11}$.

Com base na problematização apontada, lançamos como questão norteadora deste estudo: a aplicação de um protocolo de extubação será eficiente para garantir uma melhor condução do processo de extubação, diminuir os índices de insucessos, reintubações e reduzir o tempo de internação hospitalar? A motivação pela escolha desta temática se dá pela ausência e importância de um protocolo de extubação na unidade de terapia intensiva do referido hospital, onde a prática atual do processo de extubação mostra que o empirismo é insuficiente e inadequado, contrapondo-se a importância de um método padronizado que proporcione a melhor condução deste processo, sobrepondo-se ao processo de extubação empírico comumente utilizado. Buscou-se como objetivo implantar e analisar um Protocolo Operacional de Extubação na UTI adulta de um hospital de ensino na região Norte do Ceará.

\section{MÉTODO}

Trata-se de um estudo do tipo longitudinal de caráter descritivo com abordagem quantitativa para validação de um Protocolo Operacional de Extubação na UTI adulta de um hospital de ensino na região Norte do Ceará. Participaram do estudo todos os usuários internados no setor em durante o período de agosto a outubro de 2018, que se enquadraram no estudo de acordo com os critérios de inclusão e exclusão, e através de autorização do responsável legal.

Como critérios de inclusão tivemos os usuários internados na UTI Adulto do respectivo Hospital, cujo responsável legal tenha concordado em assinar o Termo de Consentimento Livre e Esclarecido e Pós-Informado, com idade igual ou maior que 18 anos, estivessem fazendo uso de VM por um período maior que $48 \mathrm{hrs,}$ apresentassem a causa da falência respiratória resolvida ou controlada; $\mathrm{PaO} 2>/$ $60 \mathrm{mmHg}$ com $\mathrm{FiO} 2</ 40 \%$ e PEEP </ 5 a $8 \mathrm{cmH} 2 \mathrm{O}$; Hemodinâmica estável, com boa perfusão tecidual, sem ou com doses baixas de vasopressores, ausência de insuficiência coronariana descompensada ou arritmias com repercussão hemodinâmica; Paciente capaz de iniciar esforços inspiratórios; Equilíbrio acidobásico e eletrolítico normais. Avaliados como aptos para iniciar o processo de desmame ventilatório e posteriormente para realização da extubação.
E como critérios de exclusão aqueles hemodinamicamente instáveis, sob uso de sedoanalgesia, que não possuíam indicação para realização de fisioterapia, aqueles cujo não se enquadrassem a realização do protocolo proposto, portadores de Síndrome do desconforto respiratório agudo (SDRA), Doença Pulmonar Obstrutiva Crônica (DPOC), e/ou Obesidade.

A prevalência em período de usuários internados nas UTI's que fazem uso de VM, onde se faz necessário a realização do processo de extubação. Foi considerado para o cálculo amostral um poder estatístico com nível de $80 \%$, com a $=5 \%$, perda amostral de 15\%. E uma redução da prevalência de insucessos da extubação e tempo demasiado do uso da VM em $20 \%$. A análise estatística se deu através de técnicas descritivas e inferências, através do uso do teste qui-quadrado (para tabelas de contingências $2 \times 2$ ), ao nível de $5 \%$ de significância (um valor-p < 0,05 é um resultado significativo indicando a decisão de se rejeitar a hipótese nula $(\mathrm{Ho})$, para cada teste aplicado neste trabalho).

A coleta de dados foi realizada por meio da aplicação de uma ficha de avaliação e com aplicação e análise de um protocolo de extubação, com realização de avaliações fisioterapêuticas diárias.

O protocolo foi elaborado pelo pesquisador e aplicado pela equipe de fisioterapia da UTI adulta do hospital em estudo, envolvendo as fases de pré-desmame, com a avaliação do paciente a partir dos critérios clínicos e indicadores fisiológicos, a fim de se tomar a decisão de iniciar o processo de desmame e posteriormente extubação; o desmame, foi realizado através do teste de respiração espontânea (TRE); e Extubação, realizada com aplicação do protocolo, com destaque para o parâmetro de sucesso no TRE, entre 30-120 minutos. Durante o TRE os pacientes foram monitorizados para sinais de insucesso descritos na tabela 2 . Foi considerado sucesso no TRE pacientes que mantiverem padrão respiratório, troca gasosa, estabilidade hemodinâmica e conforto adequados. Fazendo o uso de 
oxigênio suplementar após a retirada da ventilação superficial.

Em relação aos riscos e benefícios do estudo, o benefício está na aplicação de um protocolo de extubação com rigor científico, como um método padronizado, sobrepondo-se em relação a extubação realizada de maneira empírica, nenhuma das estratégias trouxe danos a população estudada, proporcionando assim, condutas baseadas em evidências, além da importante produção cientifica acerca do tema. Porém com a aplicação do estudo poderia o risco de falha na retirada da VM, para tanto foram utilizados índices e parâmetros para prever e identificar o melhor momento para esta conduta, identificando o momento em que o paciente estava capaz de assumir e manter a sua ventilação, quando ainda assim ocorresse o insucesso, a equipe avaliava a necessidade da realização da reintubação, realizada pelo profissional médico, para proteção das vias aéreas do paciente. Os riscos foram minimizados para proteção do participante da pesquisa; com medidas para assegurar os necessários cuidados, no caso de danos aos indivíduos e para a população estudada.

A pesquisa foi submetida e aprovada pela Comissão de Pesquisa do Departamento de Ensino e pesquisa do Hospital de En- sino Santa Casa de Misericórdia de Sobral e pelo Comitê de Ética em Pesquisa Envolvendo Seres Humanos do Hospital de Ensino Santa Casa de Misericórdia de Sobral, sob o número CAAE 89038418.6.0000.8109, sob o Parecer No 2.749.337.

\section{RESULTADOS}

O estudo foi composto por 42 pacientes, que passaram pela aplicação do protocolo de extubação orotraqueal, com idade média de 38,1 anos, de ambos os sexos, destes 29 (69\%) masculino e 13 (31\%) feminino, englobando diversas patologias de bases, e submetidos a ventilação mecânica por período maior que 48 horas. O principal achado foi que o uso de um protocolo de extubação resultou em uma redução das taxas de falha de extubação, demonstrando a eficácia do mesmo.

A figura 1 apresenta os dados de sucesso e falha no Processo de Extubação de acordo com a aplicação do protocolo havendo sucesso em 90,47\% e falha de extubação em 9,5\% da amostra, sendo que todas as falhas necessitaram retornar à ventilação mecânica em até duas horas após a extubação. A maioria dos pacientes apresentou diagnóstico de Traumatismo Cranioencefálico $(42,2 \%)$ e tempo médio de ventilação mecânica de sete dias (tabela 1).
Os pacientes que apresentaram sinais de intolerância ao processo de extubação (tabela 2), durante o período de 30 a 120 minutos de ventilação espontânea, retornaram às condições ventilatórias prévias ao desmame, sendo considerada falha do processo de extubação. Ao final de duas horas, os pacientes que não apresentaram sinais de intolerância foram considerados como sucesso no processo de extubação.

Pode-se observar que em 42 pacientes estudados, obteve-se 38 casos de sucesso e apenas 4 de falha na extubação. Na tabela 3 e 4 podemos observar o cálculo do Qui-quadrado de Pearson com valor de 11,913, razão de verossimilhança de 6,714, para significância de 0,03 (significativo ao nível de 0,05), comprovando a eficácia da utilização do protocolo de desmame na UTI.

Analisando a característica da amostra subdividida em grupo falha e grupo sucesso de extubação, observamos que a idade foi significativamente maior no grupo que necessitou de reintubação $(56,7$ anos vs 35,8 anos), não havendo diferença entre os grupos quanto ao tipo de oxigenoterapia após a extubação. Quanto ao tipo de Vias Aéreas (VVAA), todos os casos de falha evoluíram para traqueostomia. Já nos casos de sucesso, 15 pacientes encontraram-se com traqueostomia e 23 pacientes em Ar Ambiente (AA). Ob-

Figura 1 - Distribuição dos Casos de Sucesso e Falha no Processo de Extubação de acordo com a aplicação do protocolo.

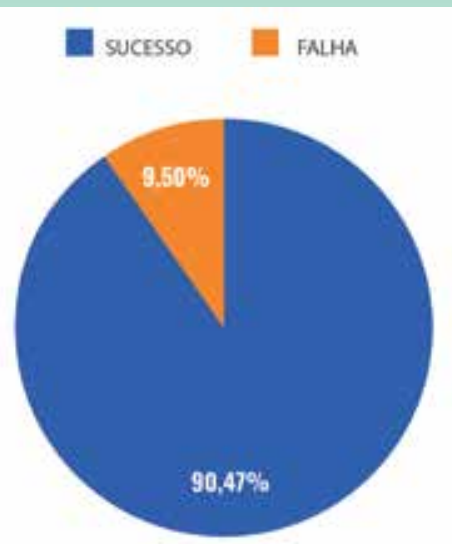

Fonte: Autoria Própria (2019). 
Tabela 1 - Diagnósticos, Idade e Índices de Sucesso e Insucesso de Extubação dos Pacientes Estudados

\begin{tabular}{lccccc|}
\hline Diagnóstico & $\begin{array}{c}\text { Idade/anos } \\
\text { (Média) }\end{array}$ & $\mathbf{N}^{\circ}$ de Pacientes & $\%$ & Sucesso & Insucesso \\
\hline Traumatismo Cranioencefálico & $18-58(30)$ & 18 & $42,2 \%$ & 15 & 1 \\
\hline Politrauma & $19-74(38)$ & 10 & $23,8 \%$ & 8 & 1 \\
\hline $\begin{array}{l}\text { Politrauma + Traumatismo Cranioen- } \\
\text { cefálico }\end{array}$ & $19-61(39)$ & 3 & $7,1 \%$ & 3 & - \\
\hline Guillian Barré & $19(19)$ & 1 & $2,3 \%$ & 1 & - \\
\hline Pancitopenia & $44(44)$ & 1 & $2,3 \%$ & 1 & - \\
\hline Acidente Vascular Encefálico & $58-62(60)$ & 2 & $4,7 \%$ & - & 2 \\
\hline Hemorragia Subaracnóidea pós-traumático & $57(57)$ & 2 & $4,7 \%$ & 2 & - \\
\hline Pós-Operatório de Laparatomia Exploratória & $66(66)$ & 1 & $2,3 \%$ & 1 & - \\
\hline Neoplasia Cerebral & $30-53(41)$ & 2 & $4,7 \%$ & 2 & - \\
\hline Complicações Pós Histerectomia & $49(49)$ & 1 & $2,3 \%$ & 1 & - \\
\hline Hemopneumotórax traumático & $18(18)$ & 1 & $2,3 \%$ & 1 & -
\end{tabular}

Fonte: Autoria Própria (2019).

Tabela 2 - Sinais de Intolerância ao teste de Respiração Espontânea

\section{Parâmetros}

Frequência respiratória

$\mathrm{SatO}_{2}$

Frequência cardíaca

Pressão arterial sistólica

Sinais e sintomas

Fonte: BRASIL ${ }^{15}$

Intolerância
$>35$ irpm
$<90 \%$
$>140 \mathrm{bpm}$
$<90>180 \mathrm{mmHg}$

Agitação, sudorese, alteração do nível de consciência

Tabela 3- Caracteristica da amostra de pacientes retirados da VM com sucesso e falha de extubação

\begin{tabular}{|c|c|c|c|}
\hline & Sucesso $n=38$ & Falha $n=4$ & $\mathrm{P}^{*}$ \\
\hline Idade (anos) & 35,8 & 56,7 & 0,685 \\
\hline Tempo de VM (dias) & 8,8 & 8,7 & 0,059 \\
\hline Tipo de VVAA & Traqueostomia $(n=15) / A A(n=23)$ & Traqueostomia & 0,434 \\
\hline Oxigenoterapia & MV a $50 \%$ & MV a $50 \%$ & - \\
\hline
\end{tabular}

Fonte: Autoria Própria (2019).

Tabela 4- Pacientes retirados da VM com sucesso e falha de extubação

\begin{tabular}{lcccc} 
& Frequência & Porcentagem & Porcentagem válida & P* \\
Sucesso & 38 & 90,5 & 90,5 & 0,031 \\
\hline Falha & 04 & 9,5 & 9,5 & - \\
Total & 42 & 100 & 100 & -
\end{tabular}

* p, nivel de significância calculado pelo teste t quiquadrado para as variáveis

Fonte: Autoria Própria (2019). 
serva-se que houve diferença mínima entre o tempo de VM (8,7 vc 8,8 dias).

As principais limitações relevantes vivenciadas neste estudo incluem principalmente: a baixa adesão dos profissionais a participarem da pesquisa, dificuldade no assentimento da equipe quanto ao protocolo proposto, o tempo reduzido para coleta de dados, pequeno tamanho da amostra, além de perdas de registros de alguns indivíduos durante a coleta de dados, e a heterogeneidade das condições clínicas da população estudada.

\section{DISCUSSÃO}

Protocolos são elaborados para que o desmame da VM se dê de forma segura e eficiente, reduzindo variações desnecessárias ou nocivas dessa abordagem. Há evidências de que, em certas circunstâncias, protocolos podem reduzir o tempo de VM. Conforme Assunção et al. 12, através de uma revisão sistemática da literatura, identificou que o uso de protocolos de desmame demonstraram em redução de $25 \%$ no tempo de VMI, de $78 \%$ na duração de desmame e de $10 \%$ no tempo de permanência na Unidade de Terapia Intensiva.

Epstein ${ }^{13}$ ressalta em seu estudo que já se sabe que a falha de extubação tem significativa associação com o aumento da mortalidade hospitalar de pacientes. Além de prolongar significativamente o tempo de VM e o tempo de permanência na UTI e no hospital, aumentando também a necessidade de traqueostomia ${ }^{14}$.

Texeira et al. ${ }^{16}$, demonstraram em seu estudo coorte que a implementação de um protocolo de extubação reduziu a taxa de reintubações em sua amostra, composta por 731 pacientes, porém sem redução no tempo de VM. Corroborando a pesquisa de Cassel et al. ${ }^{17}$ que em seu estudo sobre avaliação fisioterapêutica em uma população de pacientes críticos submetidos a um protocolo de extub-ação, observou que obteve maior sucesso em pacientes no qual foi aplicado o protocolo do que em relação aqueles que falharam e não passaram pela aplicação, apontando como principal motivo de falha a fadiga clínica, seguido de retenção de secreção e depressão do sensório, fato que vai de encontro com o referido estudo.

\section{CONCLUSÃO}

Diante do exposto, infere-se que o protocolo testado nesta pesquisa diminuiu os índices que levam a falha no processo de extubação, e consequentemente reduziu a taxa de reintubação e o tempo de internação na UTI. Vale ressaltar que protocolos devem ser elaborados e colocados em prática por equipes multiprofissionais, garantindo a integralidade na assistência aos pacientes, entretanto, protocolos jamais devem substituir ou se sobrepor o julgamento clínico, devendo ser utilizados para fim complementar.

Com isso, sugerem a necessidade de novas pesquisas, abordando um número maior de indivíduos, uso de educação permanente para a equipe multiprofissional, com a aplicação de protocolos pela equipe, a fim de poder compreender a importância da atuação do fisioterapeuta e da prática multiprofissional no processo de extubação de pacientes críticos. Pois, a partir dessa perspectiva, será possível realizar o cuidado integral, humanizado e resolutivo, oportunizando a eficácia no que diz respeito ao tratamento e reabilitação dos pacientes.

\section{Referências}

1. Silva MA, Silva VZM. Desmame da Ventilação Mecânica. Revista eletrônica saúde e ciência. 2015; 5: (1).

2. Carvalho CRR, Toufen Junior C, Franca SA. Ventilação mecânica: princípios, análise gráfica e modalidades ventilatórias. Rev J Bras Pneumol, 2009: 33: 54-70.

3. Francisco MG, Figueiredo FS, Victor EG. Análise de um Protocolo de Desmame, durante o Processo de Retirada Gradual da Ventilação Mecânica em Pacientes Internados na UTI. Criciúma: UNESC- Unidade de Terapia Intensiva do Hospital São José; 2011.

4. Presto B, Damázio L. Fisioterapia Respiratória. $4^{\mathrm{a}}$ Ed. Rio de Janeiro: Elsevier 2009.

5. Mazullo Filho JBR, Bona, S, Rosa, DP, Silva, FG, Forgiarini Junior, AL, Dias, AS, Marroni NP. Os efeitos da ventilação mecânica no estresse oxidativo. Rev. bras. ter. intensiva, 2012; 24 (1).

6. Lopes JSC, Jesus PNLG, Machado TO, Reis HFFC. Preditores De Falha De Extubação Em Unidade De Terapia Intensiva Uma Revisão De Literatura. Revista Pesquisa em Fisioterapia, 2016; 179-188.

7. Vilardo $A A B$, Oliveira VR. Gerenciamento Multidisciplinar Do Protocolo De Extubação De Ventilação Mecânica: 0 Impacto De Sua Utilização Na Extubação Programada Em Uma Uti Neonatal. São Paulo: Organização Social de saúde Santa Marcelina- Hospital Cidade Tiradentes; 2010; 24.

8. Nemer SN, Barbas CSV. Parâmetros preditivos para o desmame da ventilação mecânica. J. bras. pneumol. [online], 2011; 37(5): 669-679.

9. Moreira MF, Silva A, Bassini SRF. Incidência de falha e sucesso no processo de desmame da ventilação mecânica invasiva na Unidade de Terapia Intensiva (UTI). Rev. Cien. Linkania Júnior, 2011; 1-5.
10. Meade M, Guyatt G, Cook D, Griffith L, Sinuff T, Kergl C. Predicting success in weaning from mechanical ventilation. Rev Chest, 2011; 14: 400S-24S.

11. Macintyre NR, Cook DJ, Ely EWJR, Epstein SK, Fink JB, Heffner JE. Evidence-based guidelines for weaning and discontinuing ventilatory support: a collective task force facilitated by the American College of Chest Physicians; the American Association for Respiratory Care; and the American College of Critical Care Medicine. Rev Chest, 2011; 375S-95S.

12. Assuncao MSC, Machado FR, Rosseti HB. Avaliação de teste de tubo-T como estratégia inicial de suspensão da ventilação mecânica. Rev Brasileira de Terapia Intensiva, 2006; 18 (200):121-125.

13. Epstein SK. Weaning from ventilatory support. Curr Opin Crit Care. 2009; 15 (1): 36-43.

14. Chatburn RL, Deem S. Respiratory controversies em the critical care setting. Should weaning protocols be used with all patients who receive machenical ventilation?. Respir Care. 2007; 52 (5): 609-19.

15. Brasil. Diretrizes Brasileiras de Ventilação Mecânica - 2013. Consenso Brasileiro de Ventilação Mecânica. AMIB, 2013.

16. Texeira C, Maccari JG, Vieira SRR, Oliveira RP, Savi A, Gehm F, Borges LG, Oliveira ES. Impacto de um protocolo de desmame de ventilação mecânica na taxa de falha de extubação em pacientes de difícil desmame. J Bras Pneumol., 2012; 38 (3): $364-37$

17. Casel LW, Hahn CB, Condessa RL, Vieira SRR. Evaluation Pgysiotherapeutic Intervention in a general populations off critical patientes submitted to na arotracheal extubation protocol. Programa de Pós-Graduação em Medicina: Ciências Médicas; 2013. 